\title{
Infant Bladder Rhabdomyosarcoma in a Shared-Care Centre in Ghana: Case Report and Review of the
}

\section{Literature}

Edwin M.T. Yenli ${ }^{1,2}$, Kingsley A Bimpong ${ }^{3}$, Nihad Salifu ${ }^{4}$, Peter G. Kwarteng ${ }^{3}$, Ernest K. Cheyuo ${ }^{5}$, Yaninga H. Fuseini ${ }^{3}$, Dieu-Donnee Anyekase ${ }^{3}$, Emmanuel Akorli ${ }^{6}$, Edmund Der ${ }^{7,8}$

${ }^{1}$ Department of Surgery, Urology Unit, School of Medicine, University for Development Studies,

Box TL 1350, Tamale, Ghana

${ }^{2}$ Department of Surgery, Tamale Teaching Hospital, Box TL 1350, Tamale, Ghana

${ }^{3}$ Department of Paediatrics, Tamale Teaching Hospital, Box TL 1350, Tamale, Ghana

${ }^{4}$ Department of Paediatrics, Greater Accra Regional Hospital, Box 473, Ridge Accra, Ghana

${ }^{5}$ Tamale Teaching Hospital, Box TL 1350, Tamale, Ghana

${ }^{6}$ Department of Radiology, Tamale Teaching Hospital, Box TL 1350, Tamale, Ghana

${ }^{7}$ Department of Pathology, University for Development Studies, Box TL 1350, Tamale, Ghana

${ }^{8}$ Department of Pathology, Tamale Teaching Hospital, Box TL 1350, Tamale, Ghana

*Corresponding author: Edwin Mwintiereh Ta-ang Yenli; mwintyus@yahoo.com

Received 03 April 2021;

Accepted 16 May 2021;

Published 26 May 2021

\begin{abstract}
Rhabdomyosarcoma is a fairly common soft tissue sarcoma among children. Genitourinary rhabdomyosarcoma accounts for one tenth of rhabdomyosarcomas in the West African sub-region. However, its occurrence and management are yet to be reported in our resource limited study setting. Care of such patients could be a daunting task. A comprehensive paediatric oncology service should be advocated to optimize the chances of survival. A seven-month old boy was referred to our facility on account of posterior urethral valve. A week's complaint of crying on micturition, with associated passage of scanty urine and irritability, although he was apparently well since birth with no urinary complaints. A suprapubic mass was palpated on abdominal examination. Digital rectal examination revealed a nodular pelvic mass. Open cystostomy done and histopathology showed embryonal rhabdomyosarcoma of the urinary bladder. Chemotherapy was commenced on a modified Malignant Mesenchymal Tumour protocol of the International Society of Paediatric Oncology group. The mass was reduced by a third after six cycles of chemotherapy. In resource limited settings, the management of paediatric genitourinary rhabdomyosarcoma, using multidisciplinary and multimodal approach to include the hub for comprehensive paediatric oncology services should be advocated to optimize chances of survival
\end{abstract}

Keywords: Infant, Bladder, Rhabdomyosarcoma, Case Report, Shared-Care Center

\section{Background}

Rhabdomyosarcomas (RMS) are the commonest soft-tissue sarcomas in the paediatric population, representing about $51 \%$ of these cases, with majority being of the embryonal type ${ }^{[1]}$. It is a malignant tumour with origin from striated muscle ${ }^{[2]}$. Rhabdomyosarcomas are seen in about $15 \%$ of all solid tumours among children and it is the third most common extracranial solid neoplasm after Wilms tumour and neuroblastoma ${ }^{[3]}$. An estimate incidence of 3.9-5.2 per million, has been reported in the United States ${ }^{[4,5]}$. It has a bimodal age distribution, with peaks seen in the www.ijirms.in first five years of life and a smaller peak between the ages of 12 and $17^{[4]}$.

In Africa, RMS accounted for $5 \%$ of all paediatric cancer cases managed in Morocco ${ }^{[6]}$, with genitourinary RMS cases seen in $23 \%$ of these. It is also common in Sub-Saharan Africa as it accounted for $31 \%$ of paediatric solid tumours reported in a study in Nigeria ${ }^{[7]}$. In Ghana, a study at the Komfo Anokye Teaching hospital showed that RMS was the commonest soft tissue tumour in paediatric patients and it accounted for $5.5 \%$ of all paediatric cancer cases seen ${ }^{[8]}$. 
Genitourinary rhabdomyosarcoma accounted for $42 \%$ of paediatric rhabdomyosarcoma reported in a study from South Africa ${ }^{[9]}$, but was seen in only $10 \%$ of paediatric rhabdomyosarcoma cases reported in a study in Nigeria ${ }^{[10]}$.

Improved diagnostic techniques and management in recent years has led to improved survival ${ }^{[1,11,12]}$. In the management of RMS, two different regimens have been adopted by the American Intergroup Rhabdomyosarcoma Group (IRSG) and the International Society of Paediatric Oncology (SIOP) in Europe. In the SIOP group, chemotherapy was used to attain remission before surgery and radiotherapy for local control. However, the IRSG adopts aggressive surgery and routine radiotherapy followed by prolonged chemotherapy. In both groups, cytotoxic agents such as cyclophosphamide, vincristine, actinomycin and etoposide form an integral part of the regimens ${ }^{[13,14]}$.

Despite several reports on pediatric RMS, we were yet to see a case of genitourinary RMS in our clinical setting, therefore we aimed at reporting our experience with the management of an infant with bladder rhabdomyosarcoma in a shared-care center in a resource limited setting and reviewed the literature.

\section{Case presentation}

A seven-month old boy was referred to our facility on account of posterior urethral valve. He was apparently well since birth with no urinary complaints, until a week prior to presentation when he started crying on micturition, with associated passage of scanty urine and irritability. There was no past medical or family history of rhabdomyosarcoma. A suprapubic mass was palpated on abdominal examination. Digital rectal examination revealed a nodular pelvic mass.

Initial renal and pelvic ultrasound showed a posterior bladder wall mass protruding into the bladder cavity figure $\mathbf{1}$. There was bilateral moderate hydronephrosis on renal ultrasound. Coronal images of abdominopelvic Contrast Enhanced Computerized Tomography (CECT) scan was suggestive of bladder tumour with bilateral hydronephrosis figure 2. Serial axial images of the abdomen with intravenous contrast, revealed a heterogeneously enhancing isodense soft tissue mass in the lower part of the bladder which bridged the posterior-inferior bladder wall, extending to involve the rectovesical space figure 3 . The mass was lobulated, measured about $4.8 \times 4.9 \times 5.9 \mathrm{~cm}$ and showed a few cystic changes. No calcifications were noted. Adjacent rectum appeared normal with no evidence of infiltration. Moderate dilatation of both ureters and the pelvicalyceal systems were seen.

There was no distant metastasis on imaging, however, bilateral bone marrow aspirate was not done due to unavailability at the treatment site and inability of patient to travel for that workup at the time for various reasons. Tumour stage was T4NOM0. Baseline renal function test was unremarkable.

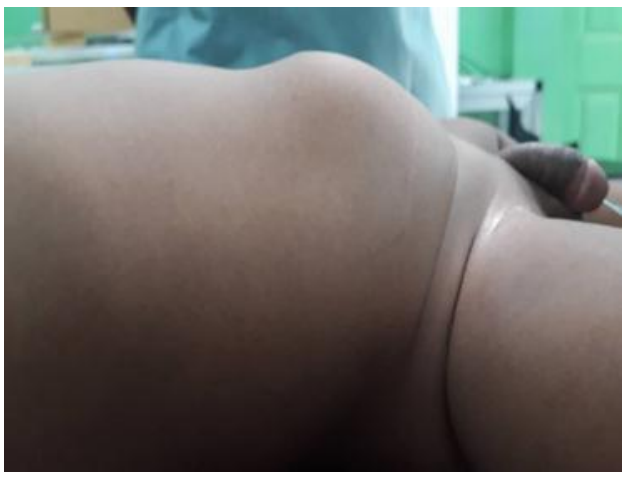

Figure 1: Physical examination. Lateral view reveals suprapubic mass
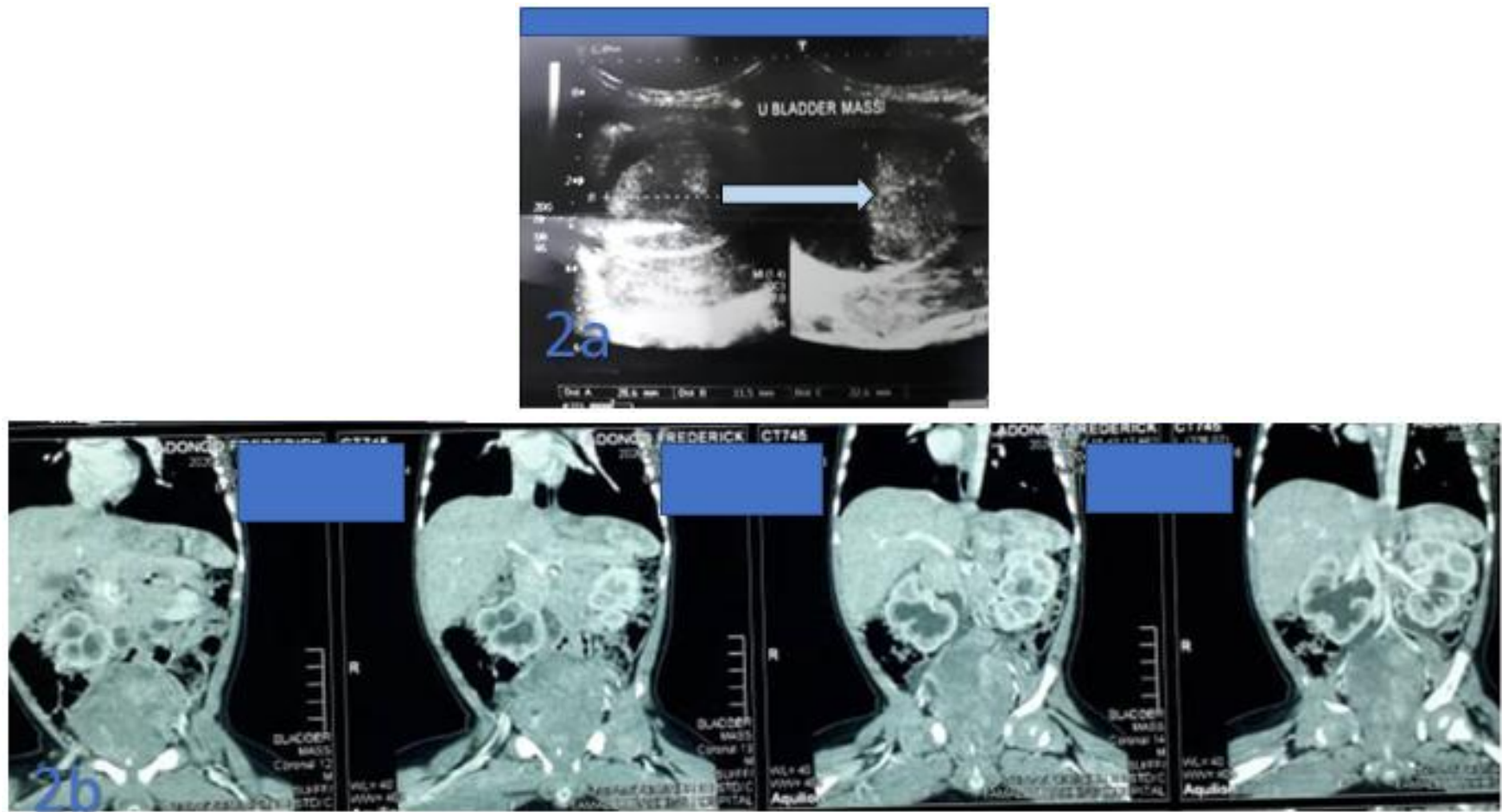

Figure 2: Imaging studies (2a) Pelvic ultrasound showing bladder tumour arrow; (2b) CECT scan showing bilateral hydronephrosis with pelvic mass prior to treatment 


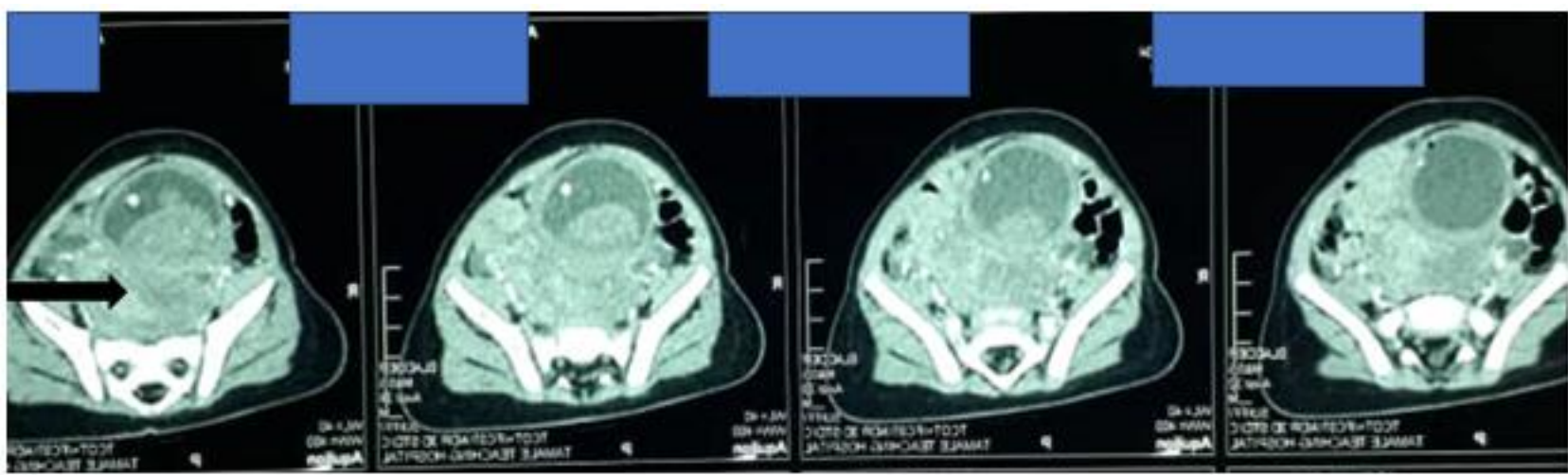

Figure 3: Pelvic CECT scan showing bladder tumour with rectovesical extension preceding treatment (arrow)

Cystostomy with upfront excision biopsy was done for emergent relief of urinary tract obstruction and the resulting hydronephrosis, with findings of multilocular mass filling up the trigone and bladder neck. The rectovesical component was not explored. No necrotic tissue was found, figure 4.
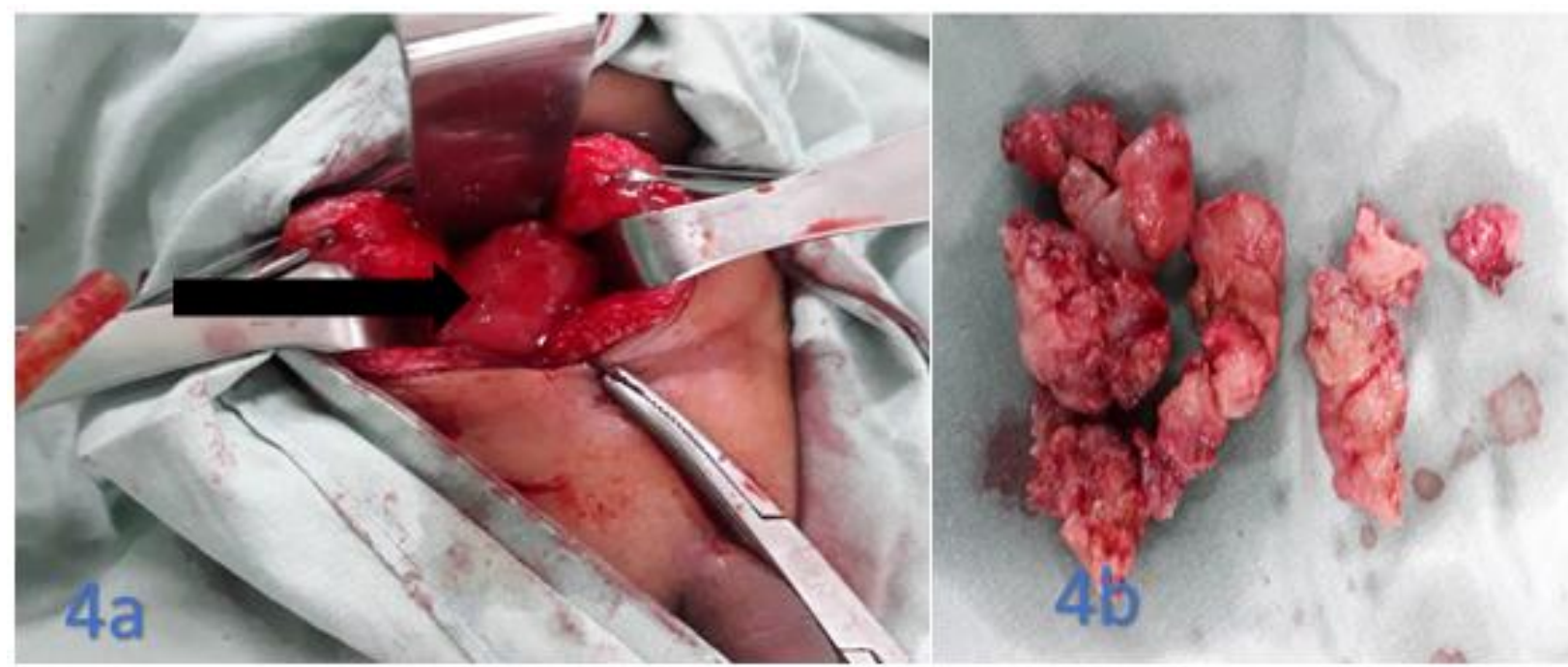

Figure 4: Intraoperative finding-(4a) bladder mass seen at cystotomy(arrow), (4b)-Specimen of resected mass for histopathology

Histopathology done showed embryonal rhabdomyosarcoma of the urinary bladder. Chemotherapy was commenced on a modified Malignant Mesenchymal Tumor protocol of the SIOP group (SIOP MMT 95) ten days after the cystostomy. The patient received three (3) cycles of Cyclophosphamide (substituted for Ifosfamide), Vincristine, Actinomycin D and Doxorubicin.

CECT scan evaluation done afterwards suggested greater than $50 \%$ partial response of the residual intraluminal bladder mass. A further three cycles of Cyclophosphamide, Vincristine, Actinomycin D were done.
A repeat CECT showed no intraluminal bladder mass, figure 5. The percentage reduction of the rectovesical mass after six (6) cycles of chemotherapy was less than a third. In view of the young age of the patient and the high incidence of late effects of radiotherapy, the possibility of second line chemotherapy was explored with the hope of achieving significant tumour response that would be amenable to complete resection or significantly reduce the radiation dose and field, if that becomes the best strategy for local control. The patient was then referred to a radiooncology center.

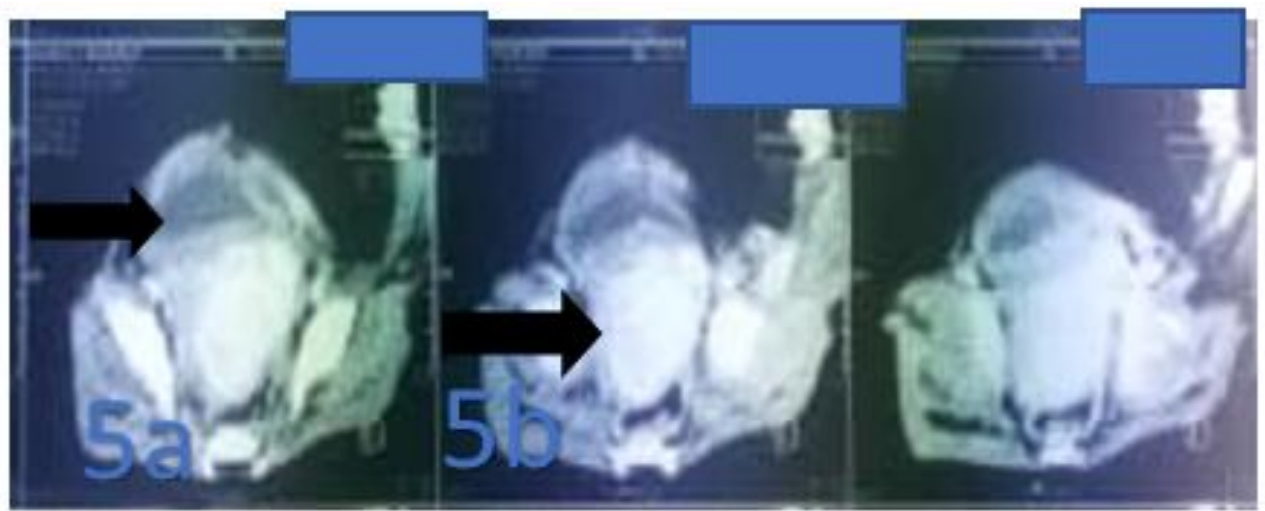

Figure 5: (5a) In a CECT scan, urinary bladder shows intact wall (arrow) with no focal mass seen. (5b) CECT scan shows mass in rectovesical pouch, no infiltration of rectum. 


\section{Discussion}

Some studies such as data analyses between 1973 and 2003 using the Surveillance, Epidemiology and End Results (SEER) database indicates that bladder cancer seems to be increasing in incidence. A male predominance with a $2: 1$ ratio has been reported ${ }^{[6,11,15]}$. Kim et al reported that rhabdomyosarcoma occurred in children below the age of 10 years ${ }^{[16]}$. In Tunisia, it accounted for greater than $50 \%$ of all soft tissue tumours and about $4 \%$ of cancers seen in the paediatric population ${ }^{[17]}$.

Our patient who was a seven months old male presented with straining, scanty urine and irritability. A palpable suprapubic mass and a nodular anterior rectal wall mass were found on examination. Like all cancers, the clinical presentation of RMS depends on the site of the primary tumour, presence of extension to surrounding structures or presence of metastasis. The common presentations of genitourinary RMS are oliguria, pain on micturition, urinary retention, hematuria, presence of abdominal mass, constipation and in rare cases extrusion of tumour tissues $[3,14,15,18,19]$. Patients may also present with an asymptomatic suprapubic mass ${ }^{[20]}$. In paediatric population, they usually present below the age of 4 years ${ }^{[3]}$. A pelvic mass may be palpated on examination ${ }^{[17,18]}$.

An initial renal and pelvic ultrasound revealed a solid posterior bladder wall tumour and bilateral hydronephrosis. The bladder tumour was staged using intravenous CECT scan which revealed a posterior bladder wall mass and invasion of the rectovesical space. In the diagnostic work up of these patients, an abdominopelvic CECT scan will be needed in addition to histopathological analysis ${ }^{[14]}$.

We performed cystostomy and resection of the tumour with the sole aim of emergent relieve of the urinary tract obstruction and to obtain sufficient tissue for analysis which is the recommended approach in such circumstances otherwise a core needle biopsy would suffice ${ }^{[20]}$. Debulking surgery is not a recommended approach in the paediatric population due to the high sensitivity of these tumours to conventional chemotherapy regimens.

The histopathology report of our patient revealed embryonal rhabdomyosarcoma. Embryonal RMS has been the most common histologic type reported in literature ${ }^{[5,6,15,21]}$, and has a higher incidence among children aged between 0 and 4 years ${ }^{[5]}$. The two main histologic subtypes are the embryonal and alveolar RMS, with the embryonal type being the most commonly reported [16,21]. Embryonal RMS may have the botryoid and spindle-cell variants ${ }^{[22]}$. A mixed botryoid and spindle cell RMS has been reported ${ }^{[19]}$. A sclerosing subtype of RMS has also been reported in the paediatric population, which presented as a sacral mass ${ }^{[23]}$. The genitourinary tract remains one of the common sites of the tumour, in addition to head, neck and extremities ${ }^{[3,14]}$. RMS involving the genitourinary tract, head and neck region are usually of the embryonal subtype whereas the alveoli subtype commonly occurs in the extremities ${ }^{[21]}$.

In the treatment of rhabdomyosarcoma, a multidisciplinary and multimodality approach is employed to include the hub for comprehensive paediatric oncology services necessary to optimize the chances of survival. In many communities in the West African Sub-region, these services may not be readily available thus posing a challenge in the care of patients with rhabdomyosarcoma. We managed this patient by engaging the expertise of colleagues at different centers. Even though this approach is not novel we have demonstrated and emphasized the need to engage other specialist in an environment with limited resources.
Preceding treatment, the patient's risks is stratified based on stage of the disease, site of tumour, histologic type and grade. Risk stratification informs the choice of modality of treatment to be selected as well as any necessary modification of management needed for patients ${ }^{[20,24]}$. Neoadjuvant chemotherapy is commenced after histopathological and radiological investigations. Our patient received the initial three (3) cycles of chemotherapy consisting of Cyclophosphamide (substituted for Ifosfamide due to lack of availability of Ifosfamide at treatment center), Vincristine, Actinomycin D and Doxorubicin, after cystostomy and biopsy had been done.

Conservative surgical resection is recommended after tumour size has been reduced with neoadjuvant chemotherapy. Afterwards, adjuvant chemotherapy or radiotherapy can be carried out based on the stage of the tumour (18). In our patient, a CECT scan evaluation after the initial three ${ }^{[3]}$ cycles suggested a greater than $50 \%$ partial response. This was then followed by another three cycles of Cyclophosphamide, Vincristine, Actinomycin D and Doxorubicin. A follow up CECT scan showed no intraluminal bladder mass, but there was an extravesical mass (percentage reduction after chemotherapy was less than a third) between bladder and rectum, for which the possibility of second line chemotherapy was considered and patient referred to a radiooncology center.

Survival in children less than one year old is known to be lower due to higher treatment-related mortality thus they receive chemotherapy doses lower than older children ${ }^{[25]}$. Also, radiotherapy for local control is usually omitted in infants because of the high incidence of late effects thus rendering overall treatment intensity in this age group suboptimal for these tumours [26-28].

\section{Conclusion}

In resource limited settings, the management of paediatric genitourinary rhabdomyosarcoma in a shared-care center using multidisciplinary and multimodal approach to include the hub for comprehensive paediatric oncology services should be advocated to optimize chances of survival.

\section{Consent for publication}

We obtained an informed consent from legal guardian of the patient to publish this report and accompanying images.

\section{List of Abbreviations}

RMS: Rhabdomyosarcomas

IRSG: Intergroup Rhabdomyosarcoma Group

SIOP: International Society of Paediatric Oncology

CECT: Contrast Enhanced Computerized Tomography

MMT: Malignant Mesenchymal Tumour

SEER: Surveillance, Epidemiology and End Results

\section{Conflict of interests}

All authors declare no conflict of interest.

\section{Funding}

No funding was received for this case report 


\section{Authors' Contribution}

EMTY conceived the idea and together with $\mathrm{KB}$, EKC drafted manuscript, NS, PK, EA, YHF, DDA, EA and ED contributed substantially to various sections of manuscript in-line with their expertise. All authors read and approved the final manuscript. All authors agree to be personally accountable for their contributions and to ensure that questions related to the accuracy or integrity of any part of the work, even ones in which the author was not personally involved, are appropriately investigated, resolved, and the resolution documented in the literature.

\section{References}

[1] Miller RW, L Young Jr J, Novakovic B. Childhood cancer. Cancer. 1995;75(S1):395-405.

[2] Leaphart C, Rodeberg D. Pediatric surgical oncology: management of rhabdomyosarcoma. Surgical oncology. 2007;16(3):173-85.

[3] Agarwala S. Pediatric rhabdomyosarcomas and nonrhabdomyosarcoma soft tissue sarcoma. Journal of Indian Association of Pediatric Surgeons. 2006;11(1):15.

[4] Ognjanovic S, Linabery AM, Charbonneau B, Ross JA. Trends in childhood rhabdomyosarcoma incidence and survival in the United States, 1975-2005. Cancer: Interdisciplinary International Journal of the American Cancer Society. 2009;115(18):4218-26.

[5] Young J, Smith M, Roffers S, Liff J, Bunin G. Cancer incidence and survival among children and adolescents: United States SEER Program 1975-1995. National Cancer Institute, SEER Program NIH. 1999.

[6] Hessissen L, Kanouni L, Kili A, Nachef MN, El Khorassani M, Benjaafar $\mathrm{N}$, et al. Pediatric rhabdomyosarcoma in Morocco. Pediatric blood \& cancer. 2010;54(1):25-8.

[7] Na'anlep MT, Echejoh GO, Manasseh NA, Mandong MB, Uba A. Paediatric solid tumours in Nigerian children: A changing pattern? African Journal of Paediatric Surgery. 2009;6(1):7.

[8] Paintsil V, Dogbe J, Blay Nguah S, Akoto A, Osei-Tutu L, Hammond C. Pattern of Childhood Cancers Presenting to the Paediatric Cancer Unit of a Tertiary Hospital in Kumasi. Journal of Cancer Prevention \&Current Research. 2015;3(3).

[9] Van Der Schyff A, Stefan DC. Clinical characteristics and outcome of rhabdomyosarcoma in South African children. African Journal Of Haematology And Oncology. 2010;1(2).

[10] Brown B, Oluwasola A. Childhood rhabdomyosarcoma in Ibadan, Nigeria: 1984-2003. Annals of tropical paediatrics. 2006;26(4):349-55.

[11] Alanee S, Shukla AR. Bladder malignancies in children aged< 18 years: results from the Surveillance, Epidemiology and End Results database. BJU international. 2010;106(4):557-60.

[12] Dagher R, Helman L. Rhabdomyosarcoma: an overview. The oncologist. 1999;4(1):34-44.

[13] Martelli H, Oberlin O, Rey A, Godzinski J, Spicer RD, Bouvet $\mathrm{N}$, et al. Conservative treatment for girls with nonmetastatic rhabdomyosarcoma of the genital tract: a report from the Study Committee of the International Society of Pediatric Oncology. Journal of clinical oncology. 1999;17(7):2117-

[14] McDowell H. Update on childhood rhabdomyosarcoma. Archives of disease in childhood. 2003;88(4):354-7.

[15] Emir S, Özdemir Sİ, Demir HA, Özyörük D, Karakus E, Tiryaki $\mathrm{T}$, et al. Pediatric bladder/prostate rhabdomyosarcoma: Eight cases from a single center. The Turkish Journal of Pediatrics. 2016;58(3):254.

[16] Kim SC, Park S, Song SH, Kim KS, Park S. Clinicopathological Characteristics of Urinary Bladder Tumors in Korean Patients 20 Years or Younger. Journal of Korean medical science. 2018;33(40).

[17] Missaoui N, Landolsi H, Jaidene L, Anjorin A, Abdelkader AB, Yaacoubi MT, et al. Pediatric rhabdomyosarcomas in Tunisia. Asian Pacific journal of cancer prevention: APJCP. 2010;11(5):1325-7.

[18] Dénes FT, Duarte RJ, Cristófani LM, Lopes RI. Pediatric genitourinary oncology. Frontiers in pediatrics. 2013;1:48.

[19] Alterio T, Chimenz R, Arena S, Conti G, Cardile S, Romeo C, et al. Mixed Botryoid and Spindle Cell Bladder Rhabdomyosarcoma: An Outstanding Pediatric Case. Case reports in pediatrics. 2017;2017.

[20] Gurria JP, Dasgupta R. Rhabdomyosarcoma and extraosseous Ewing sarcoma. Children. 2018;5(12):165.

[21] Reichek J, Barr FG. Soft tissue tumors: Rhabdomyosarcoma. Atlas of Genetics and Cytogenetics in Oncology and Haematology. 2009.

[22] Raney RB, Maurer HM, Anderson JR, Andrassy RJ, Donaldson SS, Qualman SJ, et al. The Intergroup Rhabdomyosarcoma Study Group (IRSG): major lessons from the IRS-I through IRS-IV studies as background for the current IRS-V treatment protocols. Sarcoma. 2001;5.

[23] Vadgama B, Sebire NJ, Malone M, Ramsay AD. Sclerosing rhabdomyosarcoma in childhood: case report and review of the literature. Pediatric and Developmental Pathology. 2004;7(4):391-6.

[24] Dasgupta R, Rodeberg DA, editors. Update on rhabdomyosarcoma. Seminars in pediatric surgery; 2012: Elsevier.

[25] Ragab AH, Heyn R, Tefft M, Hays DN, Newton Jr WA, Beltangady M. Infants younger than 1 year of age with rhabdomyosarcoma. Cancer. 1986;58(12):2606-10.

[26] Crist WM, Anderson JR, Meza JL, Fryer C, Raney RB, Ruymann $\mathrm{FB}$, et al. Intergroup rhabdomyosarcoma study-IV: results for patients with nonmetastatic disease. Journal of Clinical Oncology. 2001;19(12):3091-102.

[27] Ferrari A, Casanova M, Bisogno G, Zanetti I, Cecchetto $\mathrm{G}$, Bernardi BD, et al. Rhabdomyosarcoma in infants younger than one year old: a report from the Italian Cooperative Group. Cancer: Interdisciplinary International Journal of the American Cancer Society. 2003;97(10):2597-604.

[28] Joshi D, Anderson JR, Paidas C, Breneman J, Parham D, Crist W. Age is an independent prognostic factor in rhabdomyosarcoma: a report from the Soft Tissue Sarcoma Committee of the Children's Oncology Group. Pediatric blood \& cancer. 2004;42(1):64-73. 